\title{
AGRICULTURAL LAND CONVERSION IN THE SUB-URBAN AREA: A CASE STUDY OF RAJSHAHI METROPOLITAN CITY
}

\author{
Md. Abdul Halim ${ }^{1}$, Md. Mizanoor Rahman ${ }^{2 *}$ and Md. Zahidul Hassan ${ }^{3}$ \\ ${ }^{1}$ Former MS Student, Department of Geography \& Environmental Studies, University of Rajshahi \\ ${ }^{2}$ Associate Professor, Department of Geography \& Environmental Studies, University of Rajshahi \\ ${ }^{3}$ Professor, Department of Geography \& Environmental Studies, University of Rajshahi \\ *Corresponding author: e-mail: mizan_ra2005@yahoo.com
}

\begin{abstract}
Bangladesh is a land scarce country where per capita cultivated land is only 12.5 decimals. It is claimed that every year about one percent of farm land in the country is being converted to non-agricultural uses. The study is based mainly on field survey covering 2 villages (Narikel Baria and Baze Silinda) from 10 sub-urban villages around Rajshahi City Corporation under Paba Upazila of Rajshahi district. The study area was selected purposively and the respondents of this research work were selected randomly. The major focus was on to mention the general land use pattern of the area, estimates the agricultural land conversion besides determining the causes and consequences affecting such conversion. Both Narikel Baria and Baze Silinda villages were selected as study area because of its relatively higher growth in infrastructure. In order to fulfill the goal of this study, primary and secondary data were collected from various sources and to analyze and process of collected data the SPSS, Excel and Arc GIS software were used. Different types of descriptive statistics were applied for representing the analyzed data. It is observed that the land conversion form agriculture to non-agriculture allied in recent time is more than the previous time due to gradually increasing land demand in housing and relevant services which invites adverse impact on agricultural land as well as its dependent population. So, it is necessary to take integrated land-use planning to offer better options for the fulfillment of land demand both of housing and agricultural sectors.
\end{abstract}

Key word: Land use, agricultural land, land conversion, sub-urban area.

সারাংশ: বাংলাদেশ একটি কৃষি প্রধান দেশ হনেও মাথাপিছু চাষযোগ্য জমির পরিমাণ মাত্র ১২.৫ শতাংশ। প্রতি বছর কৃষি জমি অ-কৃষি জমিতে রূপান্তরিত হওয়ার মাত্রা ১ শতাংশ হারে বৃদ্ধি পাচ্ছে। শহর ও শহরতলী এলাকায় এই রূপান্তরের হার অনেক বেশি। আলোচ্য সমীক্ষায় রাজশাহী শহরের শহরতলী এলাকার সাধারণ ভূমি ব্যবহারের ধরণ, কৃষি ভূমি রূপান্তরের হার, ভূমি রপান্তরের কারণ ও ফলাফল নির্ণয় করা হয়েছে। সমীক্ষা এলাকা হিসেবে বেছে নেয়া হয়েছে পবা উপজেলার অন্ত্তগত রাজশাহী সিটি কর্পোরেশনের পার্শ্ববর্তী দুটি গ্রাম যথাঃ নারিকেলবাড়িয়া ও বাজেসিলিন্দা যা সাব-আরবান এলাকা হিসেবে পরিগনিত। সমীক্ষাটিত প্রাথমিক এবং মাধ্যমিক উভয় প্রকার তথ্য সং্्রহ ও ব্যবহার করা হয়েছে। সমীক্ষা এলাকাটি নির্ধারণের জন্য উদ্দেশ্যমূলক এবং উত্তরদাতাগণকে নির্বাচনের জন্য দৈবচয়িত নমুনায়ন পদ্ধতি ব্যবহার করা হয়েছে। সমীক্ষাটির সঠিক উদ্দেশ্যে পৌঁছানোর লক্ষ্যে সংগৃহীত তথ্যসমূহ SPSS, MS. EXCELL, ARC GIS সফ্টওয়ার এর মাধ্যমে প্রক্রিয়াজাতকরণ ও বিশ্লেষণ করা হয়েছে। বিক্লেষিত উপাত্তকে উপস্থাপনের জন্য বিভিন্ন ধরণের বর্ণনামূলক পরিসংখ্যানিক পদ্ধতি প্রয়োগ করা হয়েছে। লক্ষ্যণীয় যে, কৃষি জমি অ-কৃষি জমিতে রূপান্তরিত হওয়ার মাত্রা পূর্বের তুলনায় অনেক বেশি। মূলতঃ ক্রমাগতভাবে বসতবাড়ি এবং সংশ্লিষ্ট সেবাদানের জন্য জমির চাহিদা বৃদ্ধি কৃষি জমি এবং এর উপর নির্ভরশীল জনসংখ্যার উপর মারাত্নক প্রভাব ফেলছে। সুতরাং বসতবাড়ি এবং কৃষিভূমির চাহিদা পূরণের লক্ষ্যে সম্বন্বিত ভূমি ব্যবহার পরিকল্পনা গ্রহণ অত্যাবশ্যকীয়।

\section{Introduction}

In the last decades due to the rapid urbanization, most of the metropolitan cities of the developing countries have experienced huge increase in their population size. This trend has increased demand for land and forcing the cities to extend outward from their original demarcation. Together with the decentralization of industries, the shift of employment activities from core to sub-urban areas also has resulted in marked physical expansion of the major metropolitan cities of developing countries.

Bangladesh has always been an agrarian country where over $50 \%$ of the population is still dependent on crop agriculture (BBS, 2008). But every year Bangladesh is converting about one percent of total agricultural land from non-agriculture to agriculture. If this rate is constant, Bangladesh would convert its all arable land into non-agricultural land within next hundred years (BBC, 2010). With the growth of a country's economy, agricultural land is usually transferred to non- agriculture as the demand for non-farm products and services. This is specially so when the country's population and its per capita income rise. Transfer of farm land to non-agriculture is also needed for expansion of housing facilities in both rural and urban localities. Such transfer is also evidenced in building infrastructures such as roads, markets, educational institutions, electricity and industrial establishments, etc.

The report on Agriculture Sample Survey of Bangladesh- 2005 by Bangladesh Bureau of Statistics (BBS) shows such high rate of decline in cultivated land. Total cultivated land of all holdings in rural Bangladesh amounts to 17.77 million acres in 2005 whereas it was almost the same in 1996 before nine years. It may, however, be noted that the cultivated area per farm household has over time reduced to 1.20 acres in 2005 from 1.50 acres, recorded in 1996. This is largely due to a sharp rise in the number of rural farm households, by $24 \%$, from 11.8 million in 1996 to 14.7 million in 2005. 
Sub-urban areas have grown quickly here as poor immigrant workers moved to the inner city. The old residents, mostly whites moved to suburbs seeking better residential environments. However, when central cities and suburbs were combined into metropolitan areas, the social characteristics of suburbs greatly changed. Manufacturers moved to the suburbs, hence, a number of non-white, low-income workers also moved to these areas seeking employment (Baldassare, 1992).

Rajshahi Metropolitan City is very much in sub-urban expansion and densification of the core city situation with rapid population growth and functional expansion, which converse the agricultural land in the sub-urban area.

Land use: The term land use has been attempted to be defined differently from various perspectives by different scholars. Land use has seen as a product of interactions between a society's cultural background, skill and its physical needs in one hand and the natural potential of land on the other hand (Ram and Kolakar, 1993). Land use is also defied as men's activities on land, which are directly related to land. Land use is characterized by the arrangements, activities and inputs by people to produce change or maintain a certain land cover type (Di Gregorio and Jansen, 1998). Land use defined in this way establishes a direct link between land cover and the actions of people in their environment. Land cover is the observed (bio) physical cover on the earth's surface (Deng and others, 2009). Land cover + Land utilization $=$ Land use. According to FAO (2000) "Land use is the arrangements, activities and inputs that people undertake on a certain land cover type, According to this definitions land use reflects human activities such as the use of the land like industrial zones, residential zones, agricultural fields etc. The above definitions establish a direct link between land and the actions of people in the environment.

Agricultural Land: Land is an area of the earth surface, the characteristics of which embrace all reasonably stable, or predictably cycle, attributes of the biosphere vertically above and below this area including those of the atmosphere. 'Agricultural land' is the land base upon which agriculture is practiced. Typically, occurring on farms, agricultural activities are undertaken upon agricultural land to produce agricultural products. Kenk and others (1983) stated, "Agricultural land (also agricultural area) denotes the land suitable for agricultural production, both crops and livestock. It is one of the main resources in agriculture. In the context of zoning, agricultural land (or more properly agriculturally zoned land) refers to plots that may be used for agricultural activities, regardless of the physical type or quality of land." Agricultural land constitutes only a part of any country's territory, which in addition also includes areas not suitable for agriculture, such as forests, mountains, and inland water bodies. Agricultural land covers 38\% of the world's land area, with arable land representing less than onethird of agricultural land (11\% of the world's land area) (WDI, 2008). Agricultural characteristics, the agricultural system and the patterns in the use of agricultural lands are continuously changing in Bangladesh as well as in other parts of the world (Khan, 1989).

Land Conversion: Conversion is a normal part of urban development in both developed and developing world (Riebsame and others, 1996). Conversion means change to land use from agricultural to non-agricultural or vice versa from non-agriculture to agriculture purposes. Riebsame, et al, (1992) maintains, "The primary reason for the conversion is that under urban uses a much higher rent is recovered. The conversion from one land use to another is the activity most severely affecting terrestrial environments. The nature of conversion depends on the end use of the land." Land conversion is a phenomenon that is almost unavoidable during economic development and population growth periods. Edrijani (1994) stated, "Land conversion is a process by which land is changed from agricultural to urban uses. There is a debate on whether agricultural land fringing should be maintained or converted to other uses. This debate can be shown from both the pro-ruralist and the pro-urbanist perspectives. The pro-urbanists argue that, "land conversion is a logical consequence of urban growth. The decline of agricultural production, they argue, can be solved by intensification and technological production. Hence, land conversion is not considered as a threat in their view."

\section{Objectives}

The general objectives of this study is to identify the sub-urban area around Rajshahi Metropolitan City, patterns of sub-urban area, its causes and consequences in terms of conversion of agricultural land.

The specific objectives are as follows:

- To examine the general land use pattern in the study area;

- To analyze and detect the conversion of agricultural land of the area and

- To find out the causes and consequences of agricultural land conversion in the study area. 


\section{Materials and Methodology}

For the study both exploratory and descriptive methods have been applied to fulfill the requirement of the objectives. However two sub-urban villages were selected through simple random sampling method and the sampled villages are Narikel Baria and Baze Silinda. The standardized questionnaire survey has been carried out at the household level in the sampled villages while checklist was used for data collection from group discussion, key informants and relevant institutions. Informal group discussion was made to get information regarding different issues relevant to the study. The available project baseline survey data (secondary data) has provided relevant and useful information for an analytical study focusing on the situation of various aspects of agricultural land conversion. For analyzing collected data EXCELL and SPSS software were used for tabulation, analysis and graphic presentation. Arc GIS 9.3 and Arc View 3.2 software were also exercised for mapping analysis. To find out the similarities and differences as well as relations among different quantitative variables selected in both Metropolitan City and its sub-urban areas several tests such as chi-square test and regression analysis were applied.

\section{Study Area}

Rajshahi is the Fourth metropolitan city of Bangladesh located in between longitude $88^{\circ} 28^{\prime} \mathrm{E}$ to $88^{\circ} 38^{\prime} \mathrm{E}$ and latitude $24^{\circ} 22^{\prime} \mathrm{N}$ to $24^{\circ} 26^{\prime} \mathrm{N}$. It situated in the north western hydrological region of Bangladesh which is well known as semi-arid region in Bangladesh. Rajshahi Metropolitan City is not fully bounded over by its sub-urban area also bounded by others urban areas such as Nawhata and Katakhali Paurashava, Padma River and rural dominated areas. The sub-urban areas are situated distinctly in three different places i.e. southwest and two different locations on north side in Paba Upazila (Map 1). The south-west sub-urban area consists of 3 villages while the north-west sub-urban area contains 3 villages and north-east sub-urban area includes 4 villages. However, the total sub-urban area is located under Paba Upazila Administrative Unit, which consists of 10 villages, and one side is bounded over by the rural area of Paba Upazila whereas another side is bounded over by the Rajshahi Metropolitan City. Rajshahi Metropolitan City covers an area of approximately $48 \mathrm{~km}^{2}$ whereas the total area of suburban of Rajshahi Metropolitan City is $14.8 \mathrm{~km}^{2}$, which is about one-third of Rajshahi Metropolitan City.

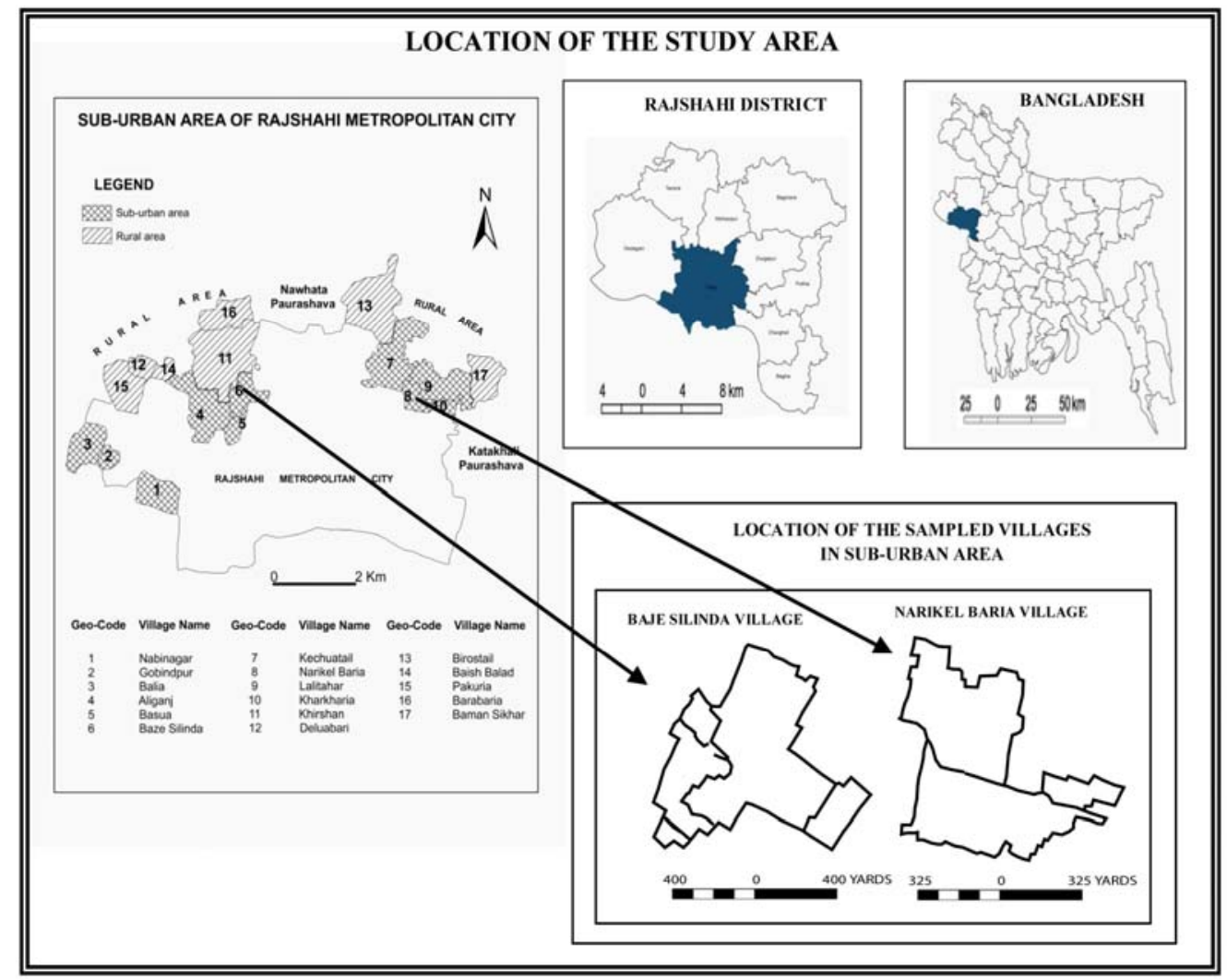

Map 1 Location of the study area. 


\section{Results and Discussion}

a. Land use pattern in the study area

As Rajshahi Metropolitan City is expanding with increasing of versatile demands of residents, so the prime agricultural land of the sub-urban area is rapidly transforming into land for housing, different orchards such as mango, lichi etc., water bodies, and roads. It represents a case study of sub-urban interface, in the phenomenon of the Rajshahi Metropolitan City urban expansion in which the area has historically played an important role in rice production but significantly replaced by the rapid urban land uses.
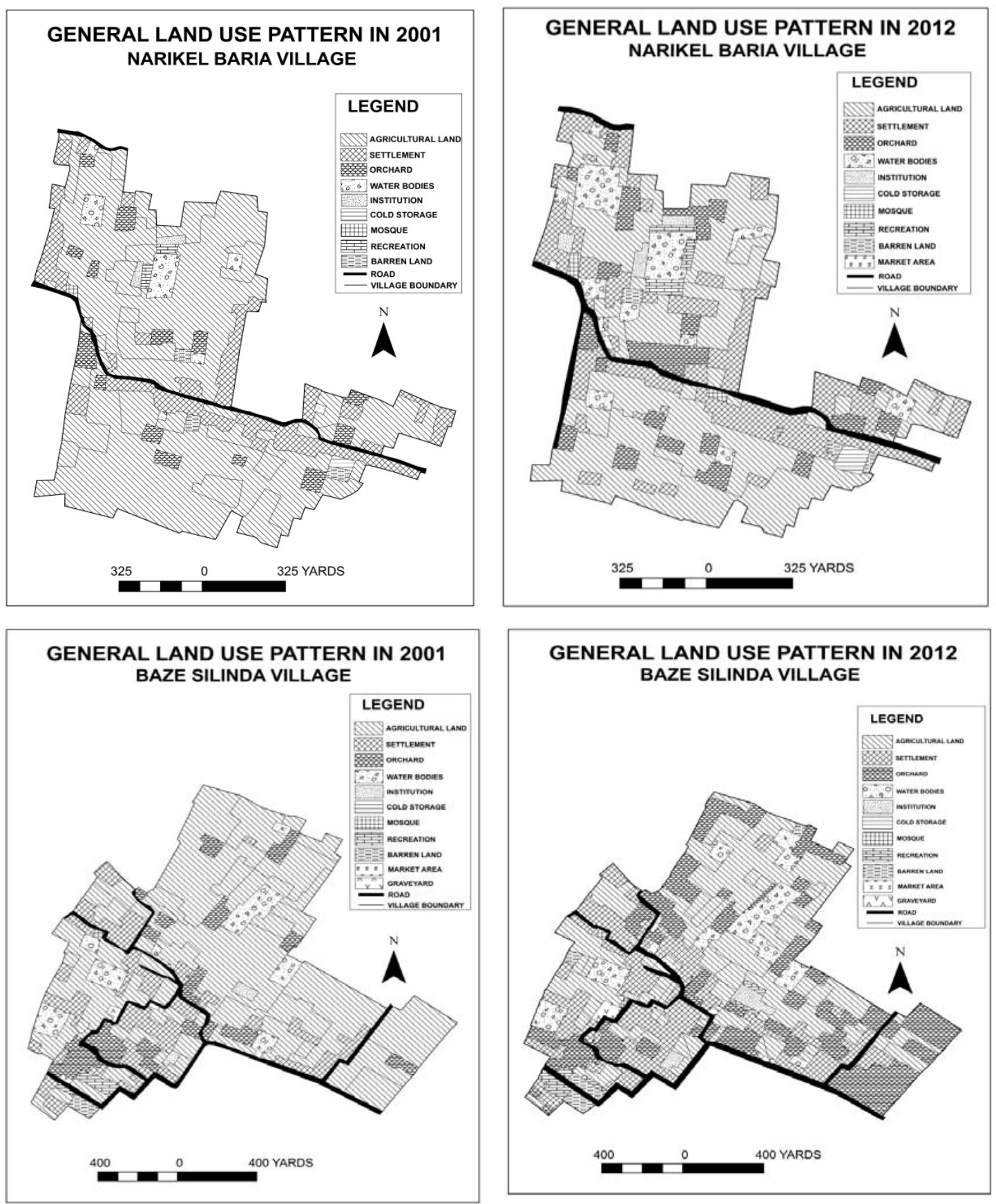

Map 2 General land use pattern of the sampled villages in sub-urban area of Rajshahi City from 2001-2012. 
Land use is the most important phenomenon because man's economic activities depend on it. It refers to the purpose the land serves, for example wild life habitat, agriculture, settlement etc. Land use deals with spatial aspects of all human activities on the land and with the way in which the land surface is adapted, or could be adopted, to serve human needs (Heimlich and others, 2001). The land use suitability is the potential capacity of a given tract of land to support different types of land utilization under given cultural and socio- economic conditions. In this study, the land use pattern from 2001-2012 has been detected carefully and the major land use categories are recognized:

- Agricultural lands : Single, double and multiple cropped area

- Settlement : Linear, circular, compact and dispersed

- Orchard: Mango, litchi, etc.

- Water bodies: Pond, fisheries, marshy land etc.

- Market area
- Institution

- Road: Pucca, semi-pucca,and kacha

- Barren land and

- Others.

\section{b. Different Types of Agricultural Land Conversion in the Study Area}

\section{Conversion of Agriculture to Agricultural Allied}

The land under agriculture was more in 2001 than 2012 both in Narikel Baria and Baze Silinda respectively, because of most of the respondents of that time were engaged in agricultural occupation and the land under infrastructure were less than 2012. Survey data indicates that, from 2001-12, among agriculture to agricultural allied the change rate of single to double cropped area has been decreased by $31.25 \%$. Single to multiple cropped areas has been decreased by $1.69 \%$ and others by $5.9 \%$. It could be noted that, double to multiple cropped areas has been increased by $4.66 \%$ (Table 1).

Table 1 Conversion of agriculture to agricultural allied in the study area

\begin{tabular}{|c|c|c|c|c|c|c|c|}
\hline \multirow[b]{2}{*}{ Conversion types } & \multicolumn{2}{|c|}{ Narikel Baria } & \multicolumn{3}{|c|}{ Baze Silinda } & \multicolumn{2}{|c|}{ Average } \\
\hline & $\begin{array}{c}2012 \\
(\%) \\
\end{array}$ & $\begin{array}{c}2001 \\
(\%) \\
\end{array}$ & $\begin{array}{c}2012 \\
(\%) \\
\end{array}$ & $\begin{array}{c}2001 \\
(\%) \\
\end{array}$ & $\begin{array}{c}2012 \\
(\%)\end{array}$ & $\begin{array}{c}2001 \\
(\%) \\
\end{array}$ & $\begin{array}{c}\text { Change } \\
2001-12(\%)\end{array}$ \\
\hline Single to double cropped area & 10.38 & 40.57 & 4.27 & 36.59 & 7.33 & 38.58 & -31.25 \\
\hline Double to multiple cropped area & 14.15 & 5.66 & 13.41 & 12.8 & 13.78 & 9.12 & +4.66 \\
\hline Single to multiple cropped area & 2.83 & 3.77 & 5.49 & 7.93 & 4.16 & 5.85 & -1.69 \\
\hline Others & 1.89 & 9.43 & 2.44 & 6.71 & 2.17 & 8.07 & -5.9 \\
\hline None & 70.75 & 40.57 & 74.39 & 35.98 & 72.57 & 38.28 & +34.29 \\
\hline Total & 100 & 100 & 100 & 100 & 100 & 100 & - \\
\hline
\end{tabular}

\section{Conversion of Agriculture to Non- agriculture}

It has already mentioned previous that during 2001-12, agricultural land rapidly has been converted into nonagriculture such as: settlement, orchard, water bodies and other purposes in the study area and this converting process is continuing.

Settlement is the most common and prominent pattern of land use pattern. Settlements in the fringe area undergo changes due to urban growth and with passage of time they are incorporated into the ever growing urban center. Field data reveals that, class-1 agricultural land has been converted more from agriculture to settlement purposes. The rate of conversion was increased by $12.2 \%$. Moreover, class- 2 has been converted by $11.43 \%$ and class- 3 by $1.25 \%$. It could be noted that, class- 4 agricultural land conversion rate to settlement was found decreased by $0.44 \%$. Whereas class- 5 has been decreased by $0.45 \%$ and class- 6 by $1.7 \%$.
Agriculture to orchard purposes, Class-1, Class-2, Class-3 and Class-4 agricultural land has been converted. Whereas class-2 agricultural land has been converted to orchard more which rate of conversion was at about $11.7 \%$, and others conversion rate are $8.26 \%, 2.93 \%$ and $1.55 \%$. In the study area most of the respondent has own orchard. Some have lease in types of orchard and some have lease out types of orchard and other types. Water bodies are the most common phenomenon in this study. Water bodies have increased than previous especially the numbers of pond have increased due to more profit.

An apparent comparison of these two sets of two different time's data indicates that, during 2001-12 agricultural land to water bodies, class- 2 agricultural land has been converted to water bodies more and the rate of conversion was $9.12 \%$. On the other hand class1 has been increased by $3.85 \%$, class- 3 by $3.72 \%$ and class-4 by $1.69 \%$. 
Agricultural land to market area Class-1, Class-2, Class-3 and Class-4 agricultural land has been converted by $5.29 \%, 5.21 \%, 1.39 \%$ and $1.55 \%$ respectively. It is observed that the conversion rate from agriculture to market use is more in class -1 and claass-2 type than other classes.

In the study area, a significant amount of agricultural land has been converted into road. It is observed that conversion rate of class- 3 and class- 4 is more than the other type and those are $1.69 \%$ and $1.24 \%$ respectively. It could also be noted that only class- 2 agricultural land conversion rate to road was decreased by $0.06 \%$ (Table 2).

Agricultural land to other types of non-agricultural land including institution: 0.05-0.1 acres of agricultural land conversion rate was increased more which is $4.33 \%$. On the other hand, $<0.05$ acres of agricultural land to recreation by $2.88 \%,<0.05$ acres of agricultural land to cold storage by $1.89 \%, 0.05-0.1$ acres of agricultural land to mosque by $0.95 \%, 0.05-0.1$ acres of agricultural land to graveyards by $0.92 \%$ and $0.05-0.1$ acres agricultural land to barren land by $1.39 \%$ (Field Survey, 2012).

Table 2 Conversion of agricultural land to non-agricultural land in the study area

\begin{tabular}{|c|c|c|c|c|c|c|c|c|c|}
\hline \multirow[b]{2}{*}{ Conversion types } & \multirow{2}{*}{$\begin{array}{l}\text { Amount of land is } \\
\text { converted (in acres) }\end{array}$} & \multirow[b]{2}{*}{ Class } & \multicolumn{2}{|c|}{ Narikel Baria } & \multicolumn{2}{|c|}{ Baze Silinda } & \multicolumn{3}{|c|}{ Average } \\
\hline & & & $\begin{array}{c}2012 \\
(\%)\end{array}$ & $\begin{array}{c}2001 \\
(\%)\end{array}$ & $\begin{array}{c}2012 \\
(\%)\end{array}$ & $\begin{array}{c}2001 \\
(\%)\end{array}$ & $\begin{array}{c}2012 \\
(\%)\end{array}$ & $\begin{array}{l}2001 \\
(\%) \\
\end{array}$ & $\begin{array}{c}\text { Change } \\
(2001-12)(\%)\end{array}$ \\
\hline \multirow{8}{*}{ Settlement } & $<0.1$ & 1 & 22.64 & 12.26 & 19.51 & 5.49 & 21.08 & 8.88 & +12.2 \\
\hline & $0.1-0.2$ & 2 & 29.25 & 9.43 & 40.24 & 18.29 & 34.75 & 13.86 & +11.43 \\
\hline & $0.21-0.3$ & 3 & 5.66 & 3.77 & 10.98 & 10.37 & 8.32 & 7.07 & +1.25 \\
\hline & $0.31-0.4$ & 4 & 3.77 & 2.83 & 4.88 & 6.71 & 4.33 & 4.77 & -0.44 \\
\hline & $0.41-0.5$ & 5 & 2.83 & 1.89 & 2.44 & 4.27 & 2.63 & 3.08 & -0.45 \\
\hline & $>0.5$ & 6 & 0.94 & 1.89 & 0 & 2.44 & 0.47 & 2.17 & -1.7 \\
\hline & No conversion & 7 & 34.91 & 67.92 & 21.95 & 52.44 & 28.43 & 60.18 & -31.75 \\
\hline & Total & & 100 & 100 & 100 & 100 & 100 & 100 & +8.26 \\
\hline \multirow{6}{*}{ Orchard } & $<0.3$ & 1 & 6.6 & 4.72 & 17.07 & 2.44 & 11.84 & 3.58 & +11.7 \\
\hline & $0.3-0.6$ & 2 & 13.21 & 5.66 & 22.56 & 6.71 & 17.89 & 6.19 & +2.93 \\
\hline & $0.61-0.9$ & 3 & 3.77 & 0.94 & 4.88 & 1.83 & 4.32 & 1.39 & +1.55 \\
\hline & $>0.9$ & 4 & 1.89 & 0 & 1.22 & 0 & 1.55 & 0 & -24.5 \\
\hline & No conversion & 5 & 74.53 & 88.78 & 54.27 & 89.02 & 64.4 & 88.9 & - \\
\hline & Total & & 100 & 100 & 100 & 100 & 100 & 100 & \\
\hline \multirow{6}{*}{ Water bodies } & $<0.1$ & 1 & 6.6 & 3.77 & 10.98 & 6.1 & 6.1 & 8.79 & +3.85 \\
\hline & $0.1-0.5$ & 2 & 11.32 & 2.83 & 18.8 & 9.15 & 9.15 & 15.11 & +9.12 \\
\hline & $0.51-1$ & 3 & 3.77 & - & 7.32 & 3.66 & 3.66 & 5.55 & +3.72 \\
\hline & $>1$ & 4 & 2.83 & 1.89 & 4.88 & 2.44 & 2.44 & 3.86 & +1.69 \\
\hline & No conversion & 5 & 75.47 & 91.51 & 57.93 & 78.66 & 78.66 & 67.7 & -17.39 \\
\hline & Total & & 100 & 100 & 100 & 100 & 100 & 100 & - \\
\hline \multirow{6}{*}{ Market area } & $<0.05$ & 1 & 15.09 & 7.55 & 6.71 & 3.66 & 10.9 & 5.61 & +5.29 \\
\hline & $0.05-0.1$ & 2 & 8.49 & 6.6 & 13.41 & 4.88 & 10.95 & 5.74 & +5.21 \\
\hline & $0.11-0.2$ & 3 & 3.77 & 2.83 & 3.66 & 1.83 & 3.72 & 2.33 & +1.39 \\
\hline & $>0.2$ & 4 & 1.89 & - & 1.83 & 0.61 & 1.86 & 0.31 & +1.55 \\
\hline & No conversion & 5 & 70.75 & 83.02 & 74.39 & 89.02 & 72.57 & 86.02 & -13.45 \\
\hline & Total & & 100 & 100 & 100 & 100 & 100 & 100 & - \\
\hline \multirow{6}{*}{ Road } & $<0.05$ & 1 & 12.32 & 7.55 & 13.41 & 17.07 & 12.86 & 12.31 & +0.55 \\
\hline & $0.05-0.1$ & 2 & 9.43 & 13.21 & 7.93 & 4.27 & 8.68 & 8.74 & -0.06 \\
\hline & $0.11-0.2$ & 3 & 5.66 & 4.72 & 5.49 & 3.05 & 5.57 & 3.88 & +1.69 \\
\hline & $>0.2$ & 4 & 3.77 & 1.89 & 1.22 & 0.61 & 2.49 & 1.25 & +1.24 \\
\hline & No conversion & 5 & 68.87 & 72.64 & 71.95 & 75.00 & 70.41 & 73.82 & -3.41 \\
\hline & Total & & 100 & 100 & 100 & 100 & 100 & 100 & - \\
\hline
\end{tabular}

\section{b. Causes of Agricultural Land Conversion}

The causes of agricultural land conversion in the study area are multifarious. Wide employment opportunity, more income and expenditure facilities attracts people to migrate in the study area which helps the area to get more population as well as rapid urban expansion indirectly. Rajshahi is very much one of the unplanned and uncontrolled urban areas of Bangladesh.
Population growth: Population growth is one of the main causes of agricultural land conversion. Figure 2 indicates that, the number of population of Narikel Baria was 609 in 1991, 889 in 2001 and 1376 in 2011. Besides the number of population of Baze Silinda was 778 in 1991, 1184 and 1553 in 2011. After that explanation the rate it is clear that, the population growth creates pressure on prime agricultural land in the study area. 


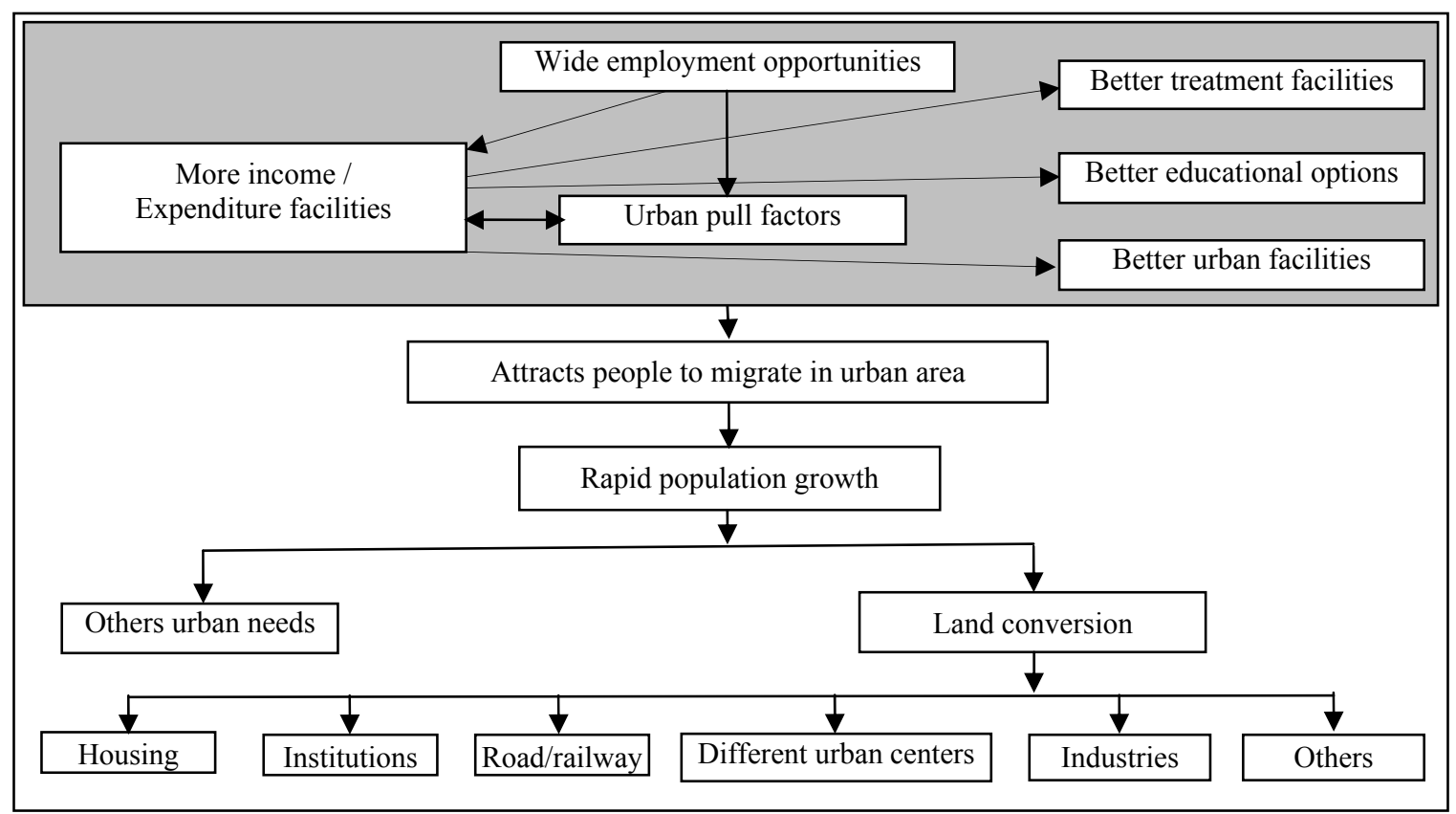

Fig. 1 Various causes influencing land conversion in sub-urban

Infrastructural development: Infrastructural development is one of the important causes closely related to population growth. In the study, emphasis putted on settlement because settlement is the main infrastructure in the study area which contributed more to land conversion than others. Due to the population growth, the infrastructure builds up quickly. To fulfill the demand of extra population, the settlement area is increasing rapidly. The number of settlements of

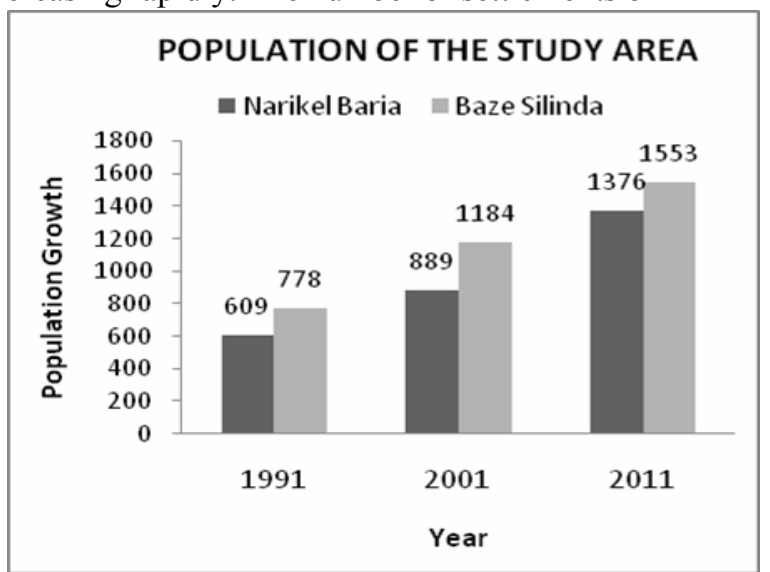

Fig. 2 Population growth in the study area Source: BBS (1991-2011)

Road is another important causes of agricultural land conversion. Rajshahi Metropolitan City is known as city of education. There are a lot of educational institutions in and around Rajshahi Metropolitan City. Among the
Narikel Baria was 113 in 1991, 144 in 2001 and 259 in 2011. On the other hand, the number of settlements of Baze Silinda was 191 in 1991, 278 in 2001 and 409 in 2011. Due to economic growth and for better living expectation many respondents had built up expensive house on agricultural land. So, the numbers of household have been increased rapidly in 2011 than 1991 and 2001 in both of the villages (Figure 3).

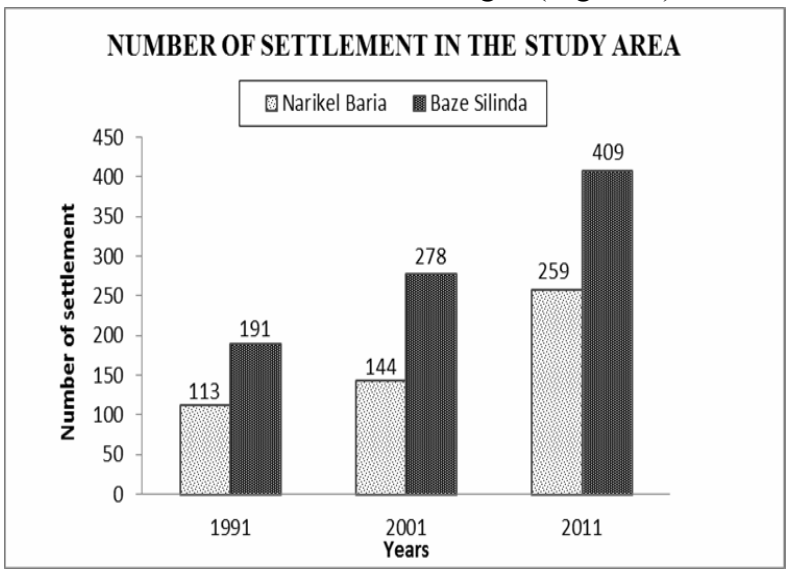

Fig. 3 Settlement growth in the study area

Source: Statistical Yearbook of Bangladesh (1991-2011)

infrastructures, the number of socio-economic institutions such as medical centers, different GOs and NGOs offices, Bank, Local Market etc are increasing gradually which occupying agricultural land. 
Communicative facilities: Communicative facilities are also the causes of agricultural land conversion in the study area. As the study area is situated surrounding Rajshahi Metropolitan City, with the development of Rajshahi Metropolitan City it's communicative especially road network has been developed dramatically by including new roads and widening existing roads which brings huge agricultural land under its networks.

More profit: The opportunity of getting large scale land in sub urban area is higher than the city area. As a result, the elite businessman purchases more land to make more profit in the near future. On the other hand, migrants from rural area have lasso's effort to reside in sub-urban area due to cheaper livelihood expenses. In the study area, it is observed from the collected data that due to more and more profit mentality of businessman and migrant's residence, every year a remarkably portion of agricultural land have been converted to diversified non-agricultural land.

Cheap and availability of land: In term of agricultural land use, sub-urban area is different than Rajshahi Metropolitan City. Most of the land of Rajshahi Metropolitan City is engaged for horticulture and farm cultivation, whereas the mixed practice is being observed in sub-urban area. So, most of the land of suburban area is cheaper and available than the Rajshahi Metropolitan City. Being cheaper and availability of agricultural land many respondents had converted their agricultural land to commercial use.

Others: Beside the above causes, there are a lot of causes of agricultural land conversion. Among them income facilities, expenditure facilities, civic facilities, taxation facilities etc. Moreover, another important cause of agricultural land conversion is expansion of Rajshahi Metropolitan City.

\section{d. Consequences Associated with Agricultural Land Conversion}

The consequences of converting agricultural land depend upon the nature of the initial and final uses of the land (Litman, 2010 b). Typically, every agricultural land has both positive and negative consequences.

Decreasing agricultural land: The most direct economic consequence of decreasing agricultural land is the farmer's loss of profits from agricultural production. Decreasing agricultural land also results in decreasing support for agriculture base businesses. These businesses are associated with different processing plants those are experiencing a direct loss when agricultural land declines.
Changing land value: In the study area, it is observed that land value is increasing gradually especially the value of closer land to the road side is more than those are far from road side. Commercial land value is more as compare to other lands. The increasing of land value in the study area is indeed the result from the potential of its location, together with the development of road networks. Moreover, in addition to the decreasing of agricultural land in the study area, the increasing land prices also enlarge the investment cost of basic infrastructure.

Occupational changes: In 2001, most of the respondents of the study area were engaged in agriculture allied occupation. But in 2012 a number of respondents had converted their agricultural land to non-agricultural purposes. As a result, they have changed their occupation especially from farmer to labor, businessman, driver, official job etc. which brings occupational diversification as well as the economic change of the study area.

Decreasing food production and rising food price: It is expected that loss of agricultural land decreases agricultural food production. For conversing of agricultural land various food productions especially food grain production and oil seeds production have been decreasing gradually. From the social point of view, most of the household members commented that, for the agricultural land conversion internal food production is decreasing and food price is hiking in the local market. Once the agro-products were available in the sub-urban area and were marketed nearby market so that the market price was very low.

Bio-diversity losses: Due to the conversion of agricultural land, farmer cultivating HYV varieties inorder to increase their production. For this, many traditional crops are almost lost their extinct. Moreover, chemical fertilizers, pesticides and insecticides etc are using for cultivation which is a cause of extinction of some environment friendly faunal species.

Environmental degradation: As per opinion of the respondents, due to decreasing agricultural land, to fulfill food demand the farmers cultivate their land intensively and use chemical fertilizer, pesticide and other poisoned elements. This is why, water, air, land and other elements of environment are being polluting day by day. Agricultural chemicals such as nitrates can threaten living things in farm fields and watercourses and can enter into drinking water reservoirs. Land can be degraded by all of the products listed as well and environment is degrading day by day. 
Others: Without the above consequences there are a lot of consequences of agricultural land conversion. Among these: changing employment opportunities, decreasing land fertility, health impact, water shortage etc.

\section{Determinants of Agricultural Land Conversion: Regression Results}

In the present study, regression analysis was carried out to identify the causes that influencing the agricultural land conversion where agricultural land conversion is the dependent value and the causes that influencing agricultural land conversion is the independent value.

e. Regression analysis between land conversion and causes of agriculture land conversion

Considering the following regression line,
$\mathrm{Y}=\beta_{0}+\beta_{1} \mathrm{X}_{1}+\beta_{2} \mathrm{X}_{2}+\beta_{3} \mathrm{X}_{3}+\beta_{4} \mathrm{X}_{4}+\beta_{5} \mathrm{X}_{5}+\beta_{6} \mathrm{X}_{6}+$ $\beta_{7} X_{7}+\beta_{8} X_{8}+\beta{ }_{9} X_{9}+\beta_{10} X_{10}+\beta_{11} X_{11}+\beta_{12} X_{12}+U$

Where, $\mathrm{Y}=$ Agricultural land conversion

$X_{1}=$ Population growth, $X_{2}=$ Infrastructural development, $\mathrm{X}_{3}=$ More profit, $\mathrm{X}_{4}=$ Communicative facilities, $\mathrm{X}_{5}=$ Institutions, $\mathrm{X}_{6}=$ Cheap and available of land, $\mathrm{X}_{7}=$ Income facilities,

$\mathrm{X} 8=$ Expenditure facilities, $\mathrm{X}_{9}=$ Civic facilities, $\mathrm{X}_{10}=$ Taxation facilities, $\mathrm{X}_{11}=$ Above all and

$\mathrm{X}_{12}=$ Others,

$\beta_{0}=$ Constant term,

$\beta 1 \ldots \beta_{12}=$ Regression co-efficient and

$\mathrm{U}=$ Random error.

Table 3 Results of Co-efficients

\begin{tabular}{|c|c|c|c|c|c|}
\hline \multirow[b]{2}{*}{ Model } & \multicolumn{2}{|c|}{ Unstandardized Co-efficients } & \multirow{2}{*}{$\frac{\text { Standardized Co-efficients }}{\text { Beta }}$} & \multirow[b]{2}{*}{$\mathrm{t}$} & \multirow[b]{2}{*}{ Sig. } \\
\hline & B & Std. Error & & & \\
\hline (Constant) & 9.918 & .234 & & 2.541 & .003 \\
\hline Population growth & 4.926 & .076 & -.064 & -.632 & .672 \\
\hline Infrastructural development & 4.831 & .134 & .341 & 3.123 & .023 \\
\hline More profit & 2.291 & .187 & .482 & 2.134 & .012 \\
\hline Communicative facilities & .902 & .034 & .741 & 14.02 & .003 \\
\hline Institution & -0.822 & .075 & .723 & 11.07 & .000 \\
\hline Cheap and available of land & .787 & .035 & .763 & 10.42 & .004 \\
\hline Income facilities & .341 & .062 & .871 & 13.45 & .458 \\
\hline Expenditure facilities & .004 & .025 & .452 & 5.73 & .043 \\
\hline Civic facilities & .005 & .094 & .237 & 6.45 & .412 \\
\hline Taxation facilities & -0.457 & .023 & .821 & 7.32 & .463 \\
\hline Above all & -0.276 & .041 & .127 & 12.46 & .237 \\
\hline Others & .452 & .025 & .452 & 4.48 & .065 \\
\hline
\end{tabular}

Finally the fitted model is, $\hat{\mathrm{Y}}=-9.918+4.926 \mathrm{X}_{1}+$ $4.831 \mathrm{X}_{2}+2.291 \mathrm{X}_{3}+0.902 \mathrm{X}_{4}-0.822 \mathrm{X}_{5}+0.787 \mathrm{X}_{6}+0.341$ $\mathrm{X}_{7}+0.004 \mathrm{X}_{8}+0.005 \mathrm{X}_{9}-0.457 \mathrm{X}_{10}-0.276 \mathrm{X}_{11}$ $+0.452 \mathrm{X}_{12}$ Where, $\mathrm{R}^{2}=0.893$ and adjusted $\mathrm{R}^{2}=0.843$.

Comment: From the above fitted model it is observed that, the effect of agricultural land conversion on population growth, infrastructural development, more profit, communicative facilities, income facilities, cheap and availability of land, expenditure facilities, above all and others are positive. On the other hand, institutions, taxation facilities and civic facilities are negative. From the information obtained the value of $R^{2}$ is 0.893 . So, it may conclude that the model explains only $89 \%$ of the total variation. The rest $(100-89)=11 \%$ depends on other causes of agricultural land conversion.

Test of regression co-efficient:

Hypothesis: $\boldsymbol{H}_{0}: \beta_{1} \ldots \ldots \ldots \ldots \ldots \ldots \ldots \ldots \beta_{12}=0$

$\boldsymbol{H}_{\mathbf{1}}: \boldsymbol{H}_{0}$ is not true.

Test statistics is, $\mathrm{F}=\{\mathrm{SSR} /(\mathrm{k}-1)\} /\{\mathrm{SSE} /(\mathrm{n}-\mathrm{k})\}$
Which is $\{(\mathrm{k}-1),(\mathrm{n}-\mathrm{k})\}$ degree of freedom and follow F-distribution

Table 4 Results of ANOVA

\begin{tabular}{cccccc}
\hline Model & $\begin{array}{c}\text { Sum of } \\
\text { squares }\end{array}$ & df & $\begin{array}{c}\text { Mean } \\
\text { square }\end{array}$ & F & Sig. \\
\hline Regression & 3.721 & 1 & 2.530 & 2.862 & 000 \\
Residual & 68.378 & 86 & .765 & & \\
\hline Total & 69.682 & 87 & & & \\
\hline
\end{tabular}

Comment: At 5\% level of significance the calculated value of $\mathbf{F}$ is 2.862 with degrees of freedom are $(1 \&$ 86) and tabulated value is 2.79 . Calculated value is greater than tabulated value. So it may reject the null hypothesis $\left(H_{0}\right)$. Therefore the test is significant. Hence, the agricultural land conversion depends on population growth, infrastructural development, more profit, communicative facilities, income facilities, cheap and availability of land, expenditure facilities, above all and others. 


\section{Key Findings}

a Agricultural land is converting gradually;

- Land conversion is more in recent time than the previous;

口 Infrastructural development especially settlement area is increasing;

More profit, income and expenditure facilities influence land conversion;

- Agricultural land is decreasing and land value is increasing gradually;

- Occupation and employment opportunities is increasing rapidly;

- Food production opportunities is decreasing and food price is hiking;

- Environmental degradation due to soil, water, air pollution through agricultural practices.

\section{Recommendations}

- The residence should be expanded vertically;

- Existing Master plan should be implemented properly to reduce the agricultural land conversion;

- High taxes can be imposed for converting agricultural land to non-agricultural purpose;

- Agricultural occupations need to be made more profitable and attractive compared to nonagriculture;

- Population growth should be controlled basically through haphazard migration ;

- People awareness should be developed through different programmes to control the agricultural land conversion;

- In addition, through introducing and proper implementation of land use regulations, specific plans, and the household permit system the situation can be improved.

\section{Conclusion}

Due to uncontrolled population growth and economic development of sub-urban area, infrastructural land has increasingly expanded which encroached upon agricultural land more than previous. The food production is decreasing, food price rising and land fertility decreasing and these all are the result of rapid agricultural land conversion. Infrastructural development is the main causes of agricultural land conversion. Though the agricultural land of the study area gradually have been decreasing so, it is necessary to implement the existing plan of RDA to arrest the magnitude of agricultural land conversion. In summary, then, the key to good development policy, in general, and food policy, in particular, is not to stop the conversion of land because of conversion is inevitable and desirable. Good policy management, however, requires that the process of conversion is done rationally and that the productivity of the remaining resources in the agricultural sector is improved. Otherwise, the agricultural land will dropout.

\section{References}

Baldassare, Mark 1992. "Suburban Communities"Annual Review of Sociology 18:475-494.

BBS, 2008, Statistical Year Book of Bangladesh, Bangladesh Bureau of Statistics, GOB, Dhaka.

BBS, 2010, Statistical Year Book of Bangladesh, Bangladesh Bureau of Statistics, GOB, Dhaka.

Deng, J S. Wand, K. Hong, Y. Qi, J.G., 2009, "Spatio-temporal dynamics and evolution of land use change and landscape pattern in response to rapid urbanization." Landscape Urban Plan. 92, pp. 187-198.

Gregorio D. and Jansen., 1998, "Defining Prime Agricultural Land and methods of Protection" Agronomy department, Purdue University.

Edrijani, 1994, "The conversion of agricultural land use and peasant marginalization in Bandung - Soreang Corridor" Undergraduate Thesis, Department of Regional and City Planning, Institute of Technology, Bandung, (in Indonesian).

FAO, 2000, "Land Cover Classification System (LCCS): Classification Concepts and User Manual," Retrieved 1-July2003, from the world wide web: http://www/fao/org./DOCREP 1003/X0596e00,htm\#p-10

Heimlich D.R., and Anderson D., 2001, "Development at the Urban Fringe and Beyond. Impacts on Agriculture and Rural Land." ERS Agricultural Economic Report No.803.

Kenk, E. and I.Cotic., April, 1983, "Land Capability Classification for Agriculture in British Columbia, MOE Manual 1 Surveys and Resource Mapping Branch, Ministry of Envirnoment and Soils Branch," Ministry of Agriculture and Food, Kelowna, B.C. 68 pp. ISSN 0821-0640.

Khan, J.R., 1989, "Changes in Agricultural landuse Pattern in Rajshahi Division 1974-1981(A study in Some Aspect of Agricultural Economy)." The Rajshahi university Studies, vol. XVII, Part-B.

Ram, B. Kolarkar, A. S., 1993, "Remote Sensing Application in Monitoring Land Use Change in Arid Rajasthan," International Journal of Remote Sensing, Vol. 3, no.7.pp.23-29.

Riebsame, W. E. H. Gosnell, and D. M. Theobald., 1996, "Land use and landscape change in the Colorado mountains, I: theory, scale, and pattern." Mountain Research and Development 16 (4): 395-405.

Riebsame., W. E. and Singh, R.B. ed., 1992, "Dynamics of Mountain Geosystems," Ashish Pub, New Delhi.

Todd Litman., 2010b, "Evaluating Transportation Economic Development Impacts," VTPI (www.vtpi.org); at www.vtpi.org/econ_dev.pdf.)

WDI -World Development Indicators online database, retrieved on July 18, 2008 (may require subscription for access; print edition from the World Bank).

Manuscript received on 23 September 2013 and revised on 12 November 2013 